\title{
Dos nuevos casos de neumonía eosinófila crónica. Revisión de la literatura
}

\author{
P. TORDERA HIGÓN, A. L. ANDREU RODRÍGUEZ, E. GÓMEZ MERINO, \\ E. PASTOR ESPLÁ, J. JIMÉNEZ RÓDENA, E. CHINER VIVES \\ Servicios de Urgencias-Observación y Radiodiagnóstico. Sección de Neumología. \\ Hospital Universitario San Juan. San Juan, Alicante
}

\author{
TWO NEW CASES OF CHRONIC EOSINOPHILIC PNEUMONIA. \\ REVISION OF THE LITERATURE
}

\section{RESUMEN}

Se presentan dos casos de neumonía eosinófila crónica en dos mujeres de 77 y 65 años. Se revisan sus características clínicas y radiológicas, así como su patogenia. Se destaca la necesidad de reconocer precozmente esta entidad así como se resalta la utilidad del lavado broncoalveolar en el diagnóstico con el fin de instaurar un tratamiento adecuado con esteroides.

PALABRAS CLAVE: Neumonía eosinófila crónica. Eosinofilias pulmonares. Enfermedad pulmonar intersticial.

\section{ABSTRACT}

We present two cases of chronic eosinophilic pneumonia in two women of a seventy-seven and sixty-five years old. We revised the clinical and radiologic features, and their pathogenesis. It si necessary to recognize in a precocious time this entity and the utility of the bronchoalveolar lavage fluid in the diagnostic, for to begin a treatment with corticosteroids.

KEY WORDS: Chronic eosinophilic pneumonia. Eosinophilic lung diseases. Interstitial lung disease.

Tordera Higón P, Andreu Rodríguez AL, Gómez Merino E, Pastor Esplá E, Jiménez Ródena J, Chiner Vives E. Dos nuevos casos de neumonía eosinófila crónica. Revisión de la literatura. An Med Interna (Madrid) 2004; 21: 391-394.

\section{INTRODUCCIÓN}

La neumonía eosinófila crónica (NEC) es una entidad poco frecuente, cuya etiología en muchas ocasiones es desconocida. Incluida inicialmente en un grupo de enfermedades que asociaban alteraciones pulmonares y eosinofilia, en 1969 Carrington y colaboradores, la definieron como una entidad propia (1). Desde el punto de vista clínico se caracteriza fundamentalmente por tos, disnea, pérdida de peso y fiebre de semanas a meses de duración radiológicamente cursa con infiltrados alveolares periféricos, presentando un porcentaje alto de eosinófilos en el lavado broncoalveolar (LBA), así como eosinofilia periférica que no siempre es constante. Su tratamiento se basa en la corticoterapia, durante un mínimo de 6 meses, por la frecuencia de recidivas (2).

Presentamos dos casos de neumonía eosinófila crónica diagnosticados en nuestro hospital, y que fueron seguidos durante al menos un año, y realizamos una revisión de esta entidad tan poco frecuente.

\section{CASOS APORTADOS}

Caso 1. Mujer de 77 años, no fumadora, que consulta por astenia, anorexia y pérdida de peso de 1 mes de evolución, asociado a febrícula, tos y disnea a mínimos esfuerzos. Se trataba de una paciente asmática de 20 años de evolución en tratamiento con beta-miméticos y corticoides inhalados, con disnea basal a moderados esfuerzos. A la exploración física presentaba $\mathrm{T}^{\mathrm{a}} \mathrm{de} 37,6{ }^{\circ} \mathrm{C}$, y a la auscultación pulmonar destacaban crepitantes de baja intensidad en campos medio e inferior izquierdo. En la analítica se objetivó una leucocitosis de $14400 / \mu$ l con un porcentaje de eosinófilos del $26 \%$, hemoglobina $14,9 \mathrm{~g} / \mathrm{dl}$, plaquetas 535.000/ml y VSG: $122 \mathrm{~mm}$ a la $1^{\mathrm{a}}$ hora, siendo el resto de parámetros normales. Presenta una gasometría arterial respirando aire ambiente con $\mathrm{pH}: 7,40, \mathrm{pO}_{2}: 54,2 \mathrm{mmHg}$, pCO2: $46 \mathrm{mmHg}, \mathrm{HCO} 3: 28 \mathrm{MM} / \mathrm{L}$ y gradiente alveolo-arterial de oxígeno de 38,3 mmHg. En la radiografía de tórax se apreció un aumento de densidad en hilio izquierdo, con infiltrados periféricos en el segmento anterior y posterior del lóbulo superior izquierdo (Fig. 1). Se inició tratamiento al ingreso con cefotaxima, eritromicina, beta-miméticos inhalados y oxigenoterapia. El tercer día de ingreso se practicó una fibrobroncoscopia por la sospecha de neumonía eosi-

Trabajo aceptado: 16 de marzo de 2004

Correspondencia: Pablo Tordera Higón. C/ El de Pagán 60, Bungalow 70. 03550 San Juan (Alicante). e-mail: pab_th@hotmail.com 


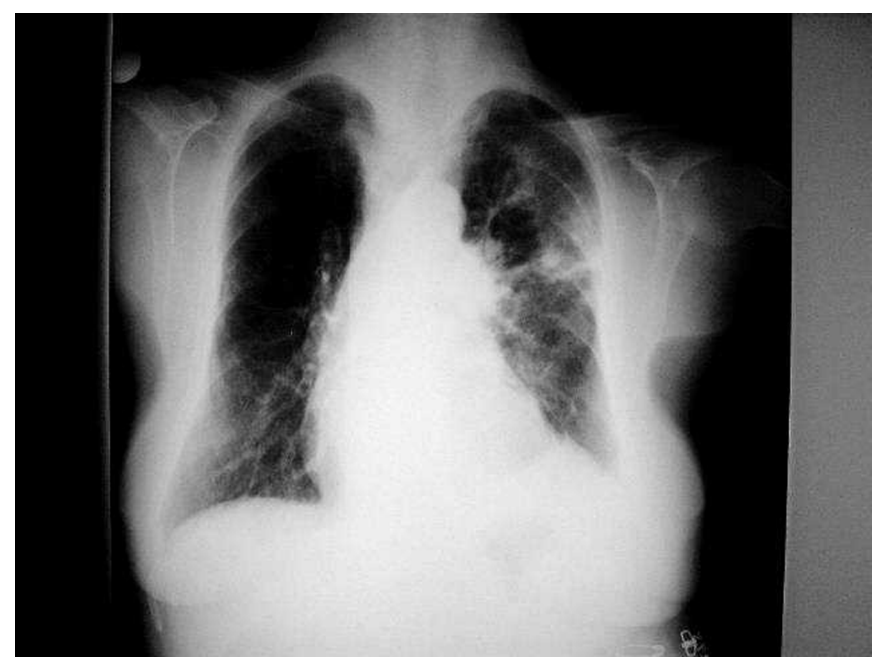

Fig. 1. Radiografía de tórax posteroanterior de la paciente número 1 que muestra infiltrados alveolares en lóbulo superior izquierdo y perihiliar izquierdo.

nófila crónica, no pudiéndose realizar LBA por mala tolerancia. En el frotis del broncoaspirado (BAS) se objetiva moderada eosinofilia. Se inició tratamiento con corticoides, objetivando una mejoría progresiva en los siguientes días y desapareciendo la eosinofilia periférica a las 24 horas de instaurar los corticoides. Al alta se remitió a su domicilio con corticoides en pauta descendente, beta-miméticos inhalados y esteroides inhalados. Al mes del alta, se suspenden los corticoides, siendo la radiografía de tórax normal y no existiendo eosinofilia periférica. Durante su seguimiento el $1^{\circ}$ año, presentó recidiva del cuadro a los 6 meses, con presencia de un $16^{\prime} 8 \%$ de eosinofilos en sangre periférica, fiebre y empeoramiento de su disnea basal. Se realiza una espirometría que mostró obstrucción severa a 1 flujo aéreo y una restricción moderada, con los siguientes parámetros: CV: $1280 \mathrm{ml}$ (60\%), FEV1 $580 \mathrm{ml}$ (37\%), CPT: $3470 \mathrm{ml}$ (79\%), Tiffenau $45(71 \%)$. Se inicia de nuevo corticoterapia que mantuvo durante casi 12 meses remitiendo la sintomatología.

Caso 2. Paciente de 65 años, no fumadora, sin antecedentes de asma bronquial, que acudió a urgencias refiriendo cuadro de fiebre vespertina, tos con escasa expectoración y disnea progresiva hasta hacerse a mínimos esfuerzos, de 1 mes de evolución. Asociaba artralgias en muñecas, astenia, anorexia y pérdida de $5 \mathrm{Kg}$ de peso. Había sido diagnosticada de neumonía 10 días antes por su médico de atención primaria, pautándose claritromicina, a pesar de lo cual empeoró el cuadro. A la exploración inicial se encontraba eupneica en reposo, con auscultación pulmonar sin hallazgos y resto de la exploración normal, salvo temperatura de $37,6^{\circ} \mathrm{C}$. En la analítica de urgencias se objetivaba una leucocitosis de $14.400 \mu / 1$ con una eosinofilia del $26,4 \%$, hemoglobina de $11,7 \mathrm{~g} / \mathrm{dl}$, plaquetas de 782.000 $\mu / 1$, PCR: 13,84 y VSG de $100 \mathrm{~mm}$ a la $1^{\text {a }}$ hora, siendo el resto de parámetros bioquímicos y la coagulación normales. La gasometría arterial respirando aire ambiente mostraba $\mathrm{pH}$ : 7,56, pO2: 67,9mmHg, pCO2: $23 \mathrm{mmHg}, \mathrm{HCO} 3: 20 \mathrm{MM} / \mathrm{L}$, saturación O2: $95 \%$ y gradiente alveolo-arterial de oxígeno $66,78 \mathrm{mmHg}$. En la radiografía de tórax presentaba infiltrados alveolares bilaterales de predominio periférico en ambos lóbulos superiores, especialmente lóbulo superior derecho pericisural y segmento 6. Se realizó TAC torácica que mostró múltiples infiltrados alveolares de distribución periférica, bilaterales, de predominio en lóbulos superiores, siendo más extensa la consolidación de lóbulo superior derecho, segmento posterior, así como infiltrado de predominio central en lóbulo superior derecho sin áreas de cavitación con pequeñas adenopatías pretraqueales y subcarinales (Fig. 2). Se inició tratamiento desde urgencias con antibioterapia de amplio espectro con ceftacidima y amikacina, asociando corticoterapia a dosis de $1 \mathrm{mg} / \mathrm{kg}$ de peso, ya que se plan-

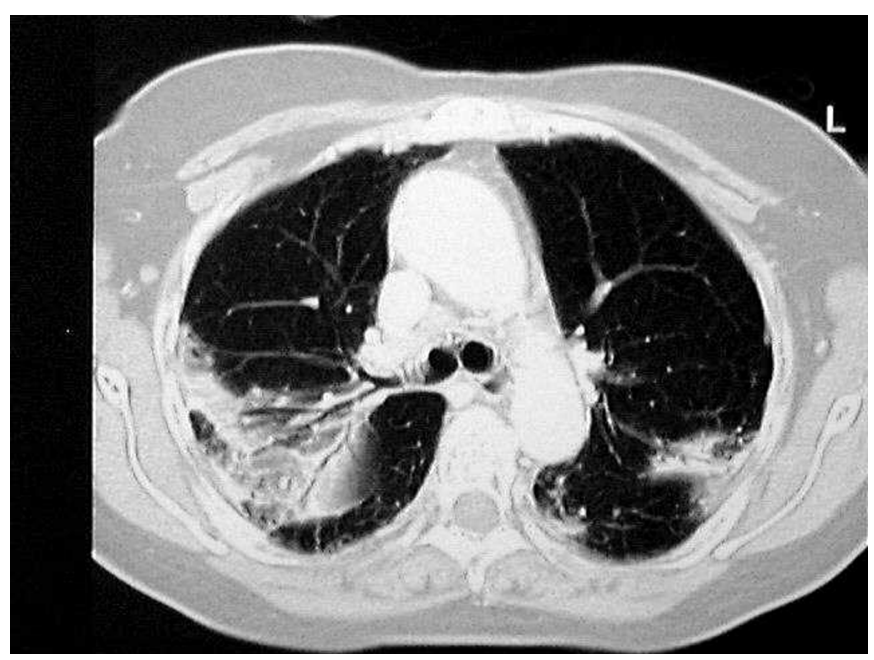

Fig. 2. TAC torácico de la paciente numero 2 que muestra infiltrados alveolo-intersticiales parcheados bilaterales.

teaba la posibilidad de que se tratara de una neumonía eosinófila crónica. A las 48 horas de su ingreso, se realizó una fibrobroncoscopia con LBA que mostró en la citometría de flujo 1400 células/microlitro con $80 \%$ de eosinófilos, así como una biopsia transbronquial que informó de ligero infiltrado crónico intersticial, con macrófagos microvacuolados en espacios alveolares con proliferación de neumocitos tipo 2 con ligera atipia citológica sin infiltrado inflamatorio eosinófilo alveolar. En 24 horas la eosinofilia periférica estaba normalizada remitiendo la fiebre y la sintomatología. En las pruebas funcionales respiratorias se objetivó una FVC: $1.980 \mathrm{ml}(84 \%)$, FEV1: $1.330 \mathrm{ml}$ (68 \%), FEV1/FVC: 67 (87\%), DLCO: 16.2 $\mathrm{ml} / \mathrm{min} / \mathrm{mmHg}(79 \%)$ y DLCO/VA: 6,26/min/mmHg (139\%). Se remitió de alta con una dosis de corticoides de $30 \mathrm{mg} / \mathrm{d}$. Al mes existía una mejoría radiográfica y espirométrica. No presentó recidivas durante el $1^{\circ}$ año de seguimiento y suspendió la corticoterapia a los 12 meses del proceso.

\section{DISCUSIÓN}

La NEC es una entidad poco frecuente de etiología desconocida. Se diferenció por primera vez de otras entidades asociadas con eosinofilia pulmonar en 1969 por Carrington et al (1) que presentaron los hallazgos existentes en 9 pacientes con una enfermedad subaguda caracterizada por síntomas sistémicos severos y respiratorios, con infiltrados pulmonares periféricos y mejoría espectacular del cuadro al tratamiento con corticoides, aunque el primer paciente diagnosticado de neumonía eosinófila crónica fue en 1960 por Christoforidis y Molnar (3). Predomina en mujeres de mediana edad, en una proporción cercana 2:1 respecto a los varones; aunque se han descrito series con una proporción similar de varones o incluso superior (4). Los pacientes afectos de NEC suelen presentar antecedentes de atopia en el 50\% de los casos, fundamentalmente asma, a menudo extrínseco $(5,6)$, aunque se puede asociar también con rinitis alérgica y pólipos nasales. Se ha visto que en pacientes asmáticos con NEC tras el diagnóstico y tratamiento parece existir un incremento en la frecuencia de las crisis. (5). En alguna ocasión se ha descrito la NEC inducida por determinados fármacos (anticomiciales, antidepresivos, antihipertensivos, anti-inflamatorios, citotóxicos, antibiótiocos,...) (7). Se ha objetivado una muy baja prevalencia de 
fumadores, un 6'5\% en la serie de Marchand et al, sin embargo se desconoce si el tabaco ejerce un efecto protector en el desarrollo de la enfermedad (6).

La NEC suele debutar como un proceso pulmonar subagudo, con síntomas de semanas a meses de duración, progresando en ocasiones a insuficiencia respiratoria grave. La forma más frecuente de presentación se basa en tos no productiva, disnea leve o moderada, fiebre y pérdida de peso, y con frecuencia, en pacientes diagnosticados previamente de asma, se puede confundir con exacerbaciones de la misma (5). En ocasiones la sospecha clínica se plantea tras el fracaso de la terapia antimicrobiana instaurada para el tratamiento de una posible neumonía de etiología infecciosa., como en nuestro caso 1. La presencia de síntomas extrarrespiratorios, a nivel de otros órganos es un raro hallazgo, debiendo así reconsiderar el diagnóstico (6). El órgano que suele presentar mayor frecuencia de afectación fuera del aparato respiratorio, es el corazón $(6,8,9)$.

En cuanto a los hallazgos de laboratorio, destaca la eosinofilia periférica, que varía según series entre un $89-95 \%$ de los pacientes $(5,6)$, requiriendo un mínimo del $6 \%$ en sangre periférica (5) o ser mayor a $1 \mathrm{~g} / \mathrm{L}$ (6). Aunque no tiene valor diagnóstico, permite monitorizar la respuesta terapéutica, pues el número de eosinófilos sanguíneos se normaliza con el tratamiento (10). No es específico de la enfermedad el aumento del número de leucocitos y aumento de la velocidad de sedimentación globular (VSG), aunque se ha relacionado el mayor número de leucocitos con mayor severidad. Se ha objetivado a su vez, que los pacientes con antecedentes de asma, muestran valores superiores de eosinófilos en sangre periférica (5). Cuando no existe eosinofilia periférica es obligado documentar eosinofilia pulmonar, ya sea por LBA o biopsia pulmonar $(5,10,11)$. Se han descrito casos con niveles elevados de $\operatorname{Ig}$ E (6), hiperglobulinemia, presencia de ANA y factor reumatoide en la NEC sin poder relacionarlos a su etiopatogenia $(12,13)$. La eosinofília en esputo podría ser útil, aunque aparece sólo en la mitad de los pacientes (5).

Respecto a las pruebas funcionales respiratorias, no existe un patrón característico en la NEC, objetivándose tanto patrones restrictivos como obstructivos, aunque estos últimos son más frecuentes en pacientes con asma. También se observa aumento del gradiente alveolo-arterial de oxígeno, hipoxemia y disminución de la capacidad de difusión en la gran mayoría de pacientes $(5,6,10)$. Estas alteraciones son menos severas de lo que se esperaría a priori dada la magnitud de la afectación radiológica (10), y suelen mejorar con el tratamiento, aunque en ocasiones puede persistir una obstrucción residual.

La radiografía de tórax se caracteriza por infiltrados alveolares periféricos en la gran mayoría de pacientes, limitados a lóbulos superiores en la mitad de los casos y siendo en un tercio, bilaterales. El patrón radiológico "negativo del edema agudo de pulmón", característico pero no patognomónico, se encuentra en la cuarta parte de los pacientes $(6,10)$ El derrame pleural no es frecuente, y cuando aparece suele ser asintomático y autolimitado $(6,14,15)$.

En el TAC son hallazgos característicos la consolidación de espacios aéreos con áreas de atenuación en vidrio deslustrado, afectando principalmente a regiones periféricas de la zona media y superior del pulmón, No apreciándose bronquiectasias como la Aspergillosis broncopulmonar alérgica (16).

La NEC puede diagnosticarse por una clínica y radiología compatibles, con o sin eosinofília periférica. Estos hallazgos, combinados con los aportados por el LBA, sustentan el diagnós- tico y pueden evitar la realización de biopsia abierta en muchas ocasiones, coviertiéndo así al LBA en una prueba con alta rentabilidad diagnóstica y con una baja morbimortalidad (10). La NEC es la principal causa de LBA con presencia de un porcentaje mayor de $40 \%$ de eosinófilos. Los linfocitos también pueden estar aumentados, aunque de forma característica el porcentaje de eosinófilos es siempre mayor que el de linfocitos, lo que ayuda a distinguirla de otras entidades en las que pudieran también estar aumentados (6). En algún caso se ha observado aumento de la relación CD4/CD8 a expensas de la disminución de CD8 (13). Se ha visto en algunas series que la eosinofilia en LBA es independiente de la existencia o no de asma (6). La biopsia transbronquial en combinación con el LBA puede ser útil, si bien las limitaciones de la técnica junto al hecho del aumento de la rentabilidad del BAL la hacen la mayoría de las ocasiones innecesaria (5). Pese a todo ello, en una cuarta parte de los pacientes, es necesario recurrir a la biopsia abierta para llegar a un diagnóstico definitivo, presentando como hallazgos más frecuentes infiltrados alveolares intersticiales con eosinófilos, histiocitos y exudado. La bronquiolitis obliterante y los microabscesos aparecen con menos frecuencia (5). En la mitad de ellos se pueden encontrar áreas focales de fibrosis intersticial (10).

Existe una serie de entidades con las cuales hay que realizar un diagnóstico diferencial, ya que se caracterizan también por infiltrados pulmonares y eosinofilia periférica o pulmonar. Entre ellas destacan fundamentalmente, la neumonía eosinófila aguda, aspergillosis broncopulmonar alérgica (ABPA), síndrome de Löffler, síndrome de Churg-Strauss y síndrome hipereosinofílico idiopático (17).

El tratamiento se fundamenta en la corticoterapia, con una mejoría espectacular y rápida, en menos de 48 horas en la gran mayoría de pacientes. La ausencia de respuesta a esteroides debe hacer replantear el diagnóstico. Si esto no sucediera, habría de plantearse diagnósticos alternativos. La respuesta radiológica completa se presenta en el $50 \%$ de los pacientes en las primeras 2 semanas (5). Las dosis iniciales eficaces están comprendidas normalmente entre 40-60 mg diarios de prednisona, y pueden ser disminuidas durante las primeras semanas, hasta una dosis de mantenimiento que varía según los pacientes, pero que suele estar comprendidas entre 5-20 mg/día (6). Se recomienda un mínimo de 6 meses de tratamiento, debido a la gran frecuencia de recaídas si se suspende el mismo. Si la enfermedad recidiva cuando se suspenden los corticoides o mientras van disminuyendo las dosis, se recomienda el tratamiento durante un año adicional antes de intentar de nuevo la retirada de corticoides. En ocasiones se ha recurrido a los esteroides inhalados para poder disminuir las dosis orales y con ello los efectos sistémicos, aunque su papel está aún por definir $(5,6)$. No es extraño que algunos pacientes requieran tratamiento durante muchos años, e incluso toda la vida, intentando mantener dosis suficientes para evitar la aparición de infiltrados y de síntomas clínicos, pero con la menor tasa posible de aparición de efectos secundarios (5). Estaría justificado con fines diagnósticos en algunos casos un tratamiento de prueba con corticoides durante 3-5 días para valorar la respuesta $(10,12,18)$.

El pronóstico de la enfermedad es en general muy bueno aunque en ocasiones se ha visto progresión de la enfermedad hacia fibrosis pulmonar (2). Este hecho, junto con la rápida respuesta a esteroides, hace patente la importancia de establecer un diagnóstico temprano para instaurar el tratamiento de forma precoz, evitando de esta manera posibles complicaciones. 


\section{Bibliografía}

1. Carrington CB, Addington WW, Goff AM, Madoff IM, Marks A, Schwaber JR, Gaensler EA. Chronic eosinophilic pneumonia. N Engl J Med 1969; 280: 787-798.

2. Allen JN, Magro CM, King MA. The eosinophilic pneumonias. Semin Respir Crit Care Med 2002; 23(2): 127-134.

3. Christoforidiasis AJ, Molnar W. Eosinophilic pneumonia: report of two cases with pulmonary biopsy. JAMA 1960; 173: 157-161.

4. Mochizuki Y, Kobashi Y, Nakahara Y, Tanaka A, Kawamura T, Sasaki S, Kawanami R. Chronic eosinophilic pneumonia - a follow study of 12 cases. Nihon Kokyuki Gakkai Zasshi 2002; 40 (11): 851-855.

5. Jederlinic PJ, Sicilian L, Gaensler EA. Chronic eosinophilic pneumonia: a report of 19 cases and a review of the literature. Medicine 1988; 67(3): 154-162.

6. Marchand E, Reynaud-Gaubert M, Lauque D, Durieu J, Tonnel A-B, Cordier J-F. Idiophatic chronic eosinophilic pneumonia: a clinical and follow-up study of 62 cases. Medicine 1998; 77(5): 299-312.

7. Dubos C, Brun J, Camus P. Etiologies medicamenteuses des pneumophaties hypereosinophiliques. References practiques actuelles; 1995: 1-8.

8. Bancal C, Sadoum D, Valeyre D, Roucou Y, Clerici C, Georges R, Battsti JP. Pneumopatie chornique idiophatique a eosinophiles. Maladie de Carrington. Presse Med 1989; 18: 1695-1698.

9. Weynants P, Riou R, Vergnon JM, Vincent M, Wiesendanger T, Cordier JF, Brune J. Pneumophaties chroniques idiophatiques a eosinophiles. Etude de 16 cas. Rev Mal Resp 1985; 2: 63-68.
10. Fernández E, Blanquer R, Domínguez A, Herrejón A, Franco J, Palop J, Plaza J. Neumonía eosinófila crónica. Análisis de 4 casos. Arch Bronconeumol 1994; 30: 414-417.

11. Gaensler EA, Carrington CB. Peripheral opacities in chronic eosinophlic pneumonia: The photographic negative of pulmonary edema. Am J Roentgenol 1977; 128: 1-13.

12. Serra J, Monserrat JM, Xaubet A, Picado C, Agustí A. Neumonía eosinófila crónica. Revisión de doce casos. Arch Bronconeumol 1986; 22 : 238-242.

13. Herrejón A, Chiner E, Blanquer R, Custardoy J, Simó M, Marín J. Neumonía eosinófila crónica. An Med Intern 1988; 5 (12): 632-634.

14. Samman YS, Wali SO, Abdelaal MA, Gangi MT, Krayem AB. Chronic eosinophilic pneumonia presenting with recurrent massive bilateral pleural effusion: case report. Chest 2001;119 (3): 968-970.

15. Rutgers SR, Schweitzer M. Chronic eosinophilic pneumonia with pleural effusion. Neth J Med 1999; 54 (5): 191-196.

16. Johkoh T, Muller NL, Akira M, Ichikado K, Suga M, Ando M, Yoshinaga $\mathrm{T}$, Kiyama $\mathrm{T}$, Mihara $\mathrm{N}$, Honda $\mathrm{O}$, Tomiyama $\mathrm{N}$, Nakamura $\mathrm{H}$. Eosinophilic lung diseases: Diagnostic accuracy of thin-section TC in 111 patients. Radiology 2000 Sept; 216(3): 773-780.

17. Pope AL, Davis WB, Allen ED, Christoforidis AJ, Allen JN. Acute eosinophilic pneumonia. A summary of 15 cases and review of the literature. Medicine 1996; 75(6): 334-342.

18. Fox B, Sead WA. Chronic eosinophilic pneumonia. Thorax 1980; 35: 570.580 . 